\title{
Going beyond the current neuroinformatics infrastructure
}

\author{
Xi Cheng ${ }^{1 *}$, Daniel Marcus ${ }^{2}$, John D. Van Horn ${ }^{3}$, Qian Luo ${ }^{4}$, Venkata S. Mattay ${ }^{1}$ and \\ Daniel R. Weinberger ${ }^{1}$
}

${ }^{1}$ Lieber Institute for Brain Development, Johns Hopkins Medical Campus, Baltimore, MD, USA, ${ }^{2}$ Department of Radiology, Washington University School of Medicine, St. Louis, MO, USA, ${ }^{3}$ Laboratory of Neuro Imaging, Department of Neurology, Institute of Neuroimaging and Informatics, University of Southern California, Los Angeles, CA, USA, ${ }^{4}$ Center for Military Psychiatry and Neuroscience Research, Walter Reed Army Research Institute, Silver Spring, MD, USA ${ }^{\dagger}$

Keywords: neuroinformatics, infrastructure, neuroscience, informatics, data sharing

\section{OPEN ACCESS}

Edited and reviewed by: Daniel Gardner,

Weill Cornell Medical College, USA

*Correspondence: Xi Cheng, chengxi001@gmail.com

${ }^{\dagger}$ The opinions or assertions contained herein are the private views of the author, and are not to be construed as official, or as reflecting true views of the Department of the Army or the Department of Defense.

Received: 28 April 2015 Accepted: 27 May 2015 Published: 16 June 2015

Citation: Cheng X, Marcus D, Van Horn JD, Luo Q, Mattay VS and Weinberger DR (2015) Going beyond the current neuroinformatics infrastructure.

Front. Neuroinform. 9:15. doi: 10.3389/fninf.2015.00015
The enormous volume of multi-modal neuroimaging data across different neuroscience research communities poses a daunting challenge to traditional methods of data sharing, data archiving, data processing, and data analysis (Van Horn and Toga, 2014).

Neuroinformatics plays a crucial role in creating advanced methodologies and tools for the handling of varied and heterogeneous datasets in order to better understand the structure and function of the brain. These tools and methodologies not only enhance data collection, analysis, integration, interpretation, modeling, and data dissemination, but also promote data sharing and collaboration (Cox, 1996; Smith et al., 2004; Friston, 2006; Marcus et al., 2007; Dinov et al., 2009; Van Horn and Toga, 2009) which are essential elements for making progress efficiently in this rapidly burgeoning field.

The purpose of this special issue is to use case studies of the state-of-art neuroinformatics infrastructure to anticipate and project future generation systems.

A number of leading research groups from different parts of the world were invited to participate in this research topic. Each of the contributions provided a showcase solution to domain specific challenges we currently face. We will try to review these articles according to the categories of the issues they covered. Some articles covered multiple categories. However, due to the limited space, we only discuss them under one category.

Articles by Bartsch et al. (2014), Goscinski et al. (2014), Haselgrove et al. (2014), King et al. (2014), Marenco et al. (2014), Muehlboeck et al. (2014), Rane et al. (2014), Rautenberg et al. (2014), Sherif et al. (2014), and Wood et al. (2014), present solutions for data archiving and related issues including, how to efficiently collect, store, query, visualize and share large volume neuroimaging data. Some of these systems are large in scale, geographically distributed, and already have a large dataset and a well-established user community.

Beyond neuroimaging, Sobolev et al. (2014) present a data management platform for neurophysiological data, and Mouček et al. (2014), and Tripathy et al. (2014) describe techniques and methodologies for collecting and managing electrophysiological data.

Once the incoming data have been archived, there are many other important issues that need to be addressed.

First, how to visualize the data to meet domain-specific needs is still an open-ended research question. Gutman et al. (2014) present a light framework to visualize DICOM images stored in the Extensible Neuroimaging Archive Toolkit (XNAT). Hänel et al. (2014) describe an application with two designs for the 3D visualization of the human brain.

Second, how to efficiently process huge volumes of datasets is challenging especially when bottom-up explorative data analysis becomes more and more popular. Contributions from Andronache et al. (2013), Da Mota et al. (2014), Dinov et al. (2014), Eklund et al. (2014), Friedel et al. (2014), and Mahmud et al. (2014), discuss opportunities and methodologies that facilitate 
large-scale parallel data processing tasks under a heterogeneous computational environment.

Third, how to mine the data i.e., how to extract meaningful information from the data, is the most challenging part of all. Liu and Calhoun (2014) provide a review of multivariate analyses approaches in Imaging Genetics. Goh et al. (2014) discuss challenges in neuroinformatics of Traumatic Brain Injury neuroimaging analysis in the context of structural, connectivity, and functional paradigms. The manuscript by Miller et al. (2013) describes novel neuroinformatics technologies at $1 \mathrm{~mm}$ anatomical scale based on high-throughput 3D functional and structural imaging technologies of the human brain. Xiang et al. (2014) explored novel data analysis methodologies and platforms for handling large volumes of neuromagnetic data with a very wide range of temporal frequencies. Kauppi et al. (2014), introduce a versatile software package for inter-subject correlation based analyses of fMRI data.

Finally, there are a number of contributions discussing other topics important to the neuroinformatics infrastructure. Zaslavsky et al. (2014) describe a prototype implementation of digital atlasing infrastructure initiated by the International Neuroinformatics Coordinating Facility (INCF). Herrick et al. (2014) showcase how to use dictionary service to extend metadata

\section{References}

Andronache, A., Rosazza, C., Sattin, D., Leonardi, M., D’Incerti, L., and Minati, L. (2013). Impact of functional MRI data preprocessing pipeline on defaultmode network detectability in patients with disorders of consciousness. Front. Neuroinform.7:16. doi: 10.3389/fninf.2013.00016

Bartsch, H., Thompson, W. K., Jernigan, T. L., and Dale, A. M. (2014). A webportal for interactive data exploration, visualization, and hypothesis testing. Front. Neuroinform. 8:25. doi: 10.3389/fninf.2014.00025

Cox, R. W. (1996). AFNI: software for analysis and visualization of functional magnetic resonance neuroimages. Comput. Biomed. Res. 29, 162-17310.1006/cbmr.1996.0014

Da Mota, B., Tudoran, R., Costan, A., Varoquaux, G., Brasche, G., Conrod, P., et al. Consortium (2014). Machine learning patterns for neuroimaging-genetic studies in the cloud.Front. Neuroinform. 8:31. doi: 10.3389/fninf.2014.00031

Das, S., McCaffrey, P. G., Talkington, M. W. T., Andrews, N. A., Corlosquet, S., Ivinson, A. J., et al. (2014). Pain Research Forum: application of scientific social media frameworks in neuroscience. Front. Neuroinform. 8:21. doi: 10.3389/fninf.2014.00021

Dinov, I. D., Petrosyan, P., Liu, Z., Eggert, P., Hobel, S., Vespa, P., et al. (2014). High-throughput neuroimaging-genetics computational infrastructure. Front. Neuroinform. 8:41. doi: 10.3389/fninf.2014.00041

Dinov, I. D., Van Horn, J. D., Lozev, K. M., Magsipoc, R., Petrosyan, P., Liu, Z., et al. (2009). Efficient, distributed and interactive neuroimaging data analysis using the LONI Pipeline. Front. Neuroinform. 3:22. doi: 10.3389/neuro.11.022.2009

Eklund, A., Dufort, P., Villani, M., and LaConte, S. (2014). BROCCOLI: software for fast fMRI analysis on many-core CPUs and GPUs. Front. Neuroinform. 8:24. doi: 10.3389/fninf.2014.00024

Evans, R. C., and Polavaram, S. (2013). Growing a garden of neurons. Front. Neuroinform. 7:17. doi: 10.3389/fninf.2013.00017

Friedel, M., van Eede, M. C., Pipitone, J., Chakravarty, M. M., and Lerch, J. P. (2014). Pydpiper: a flexible toolkit for constructing novel registration pipelines. Front. Neuroinform. 8:67. doi: 10.3389/fninf.2014.00067

Friston, K. J. (2006). Statistical Parametric Mapping. London: Academic Press

Goh, S. Y. M., Irimia, A., Torgerson, C. M., and Van Horn, J. D. (2014). Neuroinformatics challenges to the structural, connectomic, functional, across XNAT database instances. Sarwate et al. (2014) review the relevant literature on differential privacy, a framework for measuring and tracking privacy loss in these settings, and demonstrate the feasibility of using this framework to calculate statistics on data distributed at many sites while still providing privacy. Das et al. (2014) report a case study on how to foster discussion and communication by using an open-source content management system. Evans and Polavaram (2013) provide a general commentary article in the field of computational models of biologically realistic neuronal networks.

We intend this Special Issue as more than a compendium of current systems. We wish to stimulate on-going discussions at the level of the neuroinformatics infrastructure including: -what are the common challenges the next generation of infrastructure will have to address? - what new technologies will be of maximum benefit? -how will we go beyond the limits of the current generation infrastructure? and -what are the key features next generation infrastructure should implement? Such discussions will inspire and help guide the development of a state of the art, highly-efficient neuroinformatics infrastructure. Such research community wide productive catalytic reactions will be a testament to the worthiness of our efforts in creating this Special Issue.

and electrophysiological multimodal imaging of human traumatic brain injury.Front. Neuroinform. 8:19. doi: 10.3389/fninf.2014.00019

Goscinski, W. J., McIntosh, P., Felzmann, U., Maksimenko, A., Hall, C. J., Gureyev, T., et al. (2014). The multi-modal Australian ScienceS Imaging and Visualization Environment (MASSIVE) high performance computing infrastructure: applications in neuroscience and neuroinformatics research. Front. Neuroinform. 8:30. doi: 10.3389/fninf.2014.00030

Gutman, D. A., Dunn, W. D. Jr., Cobb, J., Stoner, R. M., Kalpathy-Cramer, J., and Erickson, B. (2014). Web based tools for visualizing imaging data and development of XNATView, a zero footprint image viewer. Front. Neuroinform. 8:53. doi: 10.3389/fninf.2014.00053

Hänel, C., Pieperhoff, P., Hentschel, B., Amunts, K., and Kuhlen, T. (2014). Interactive $3 \mathrm{D}$ visualization of structural changes in the brain of a person with corticobasal syndrome. Front. Neuroinform. 8:42. doi: 10.3389/fninf.2014.00042

Haselgrove, C., Poline, J.-B., and Kennedy, D. N. (2014). A simple tool for neuroimaging data sharing. Front. Neuroinform. 8:52. doi: 10.3389/fninf.2014.00052

Herrick, R., McKay, M., Olsen, T., Horton, W., Florida, M., Moore, C. J., et al. (2014). Data dictionary services in XNAT and the Human Connectome Project. Front. Neuroinform. 8:65. doi: 10.3389/fninf.2014.00065

Kauppi, J.-P., Pajula, J., and Tohka, J. (2014). A versatile software package for inter-subject correlation based analyses of fMRI.Front. Neuroinform. 8:2. doi: 10.3389/fninf.2014.00002

King, M. D., Wood, D., Miller, B., Kelly, R., Landis, D., Courtney, W., et al. (2014). Automated collection of imaging and phenotypic data to centralized and distributed data repositories. Front. Neuroinform. 8:60. doi: 10.3389/fninf.2014.00060

Liu, J., and Calhoun, V. D. (2014). A review of multivariate analyses in imaging genetics. Front. Neuroinform. 8:29. doi: 10.3389/fninf.2014.00029

Mahmud, M., Pulizzi, R., Vasilaki, E., and Giugliano, M. (2014). QSpike tools: a generic framework for parallel batch preprocessing of extracellular neuronal signals recorded by substrate microelectrode arrays. Front. Neuroinform. 8:26. doi: 10.3389/fninf.2014.00026

Marcus, D. S., Olsen, T. R., Ramaratnam, M., and Buckner, R. L. (2007) The Extensible Neuroimaging Archive Toolkit: an informatics platform for 
managing, exploring, and sharing neuroimaging data. Neuroinformatics 5, 11-34. doi: 10.1385/NI:5:1:11

Marenco, L. N., Wang, R., Bandrowski, A. E., Grethe, J. S., Shepherd, G. M., and Miller, P. L. (2014). Extending the NIF DISCO framework to automate complex workflow: coordinating the harvest and integration of data from diverse neuroscience information resources.Front. Neuroinform. 8:58. doi: 10.3389/fninf.2014.00058

Miller, M. I., Faria, A. V., Oishi, K., and Mori, S. (2013). High-throughput neuroimaging informatics. Front. Neuroinform. 7:31. doi: 10.3389/fninf.2013.00031

Mouček, R., Ježek, P., Vařeka, L., Řondík, T., Brůha P, Papež V., Mautner, P., et al. (2014). Software and hardware infrastructure for research in electrophysiology. Front. Neuroinform. 8:20. doi: 10.3389/fninf.2014.00020

Muehlboeck, J.-S., Westman, E., and Simmons, A. (2014). TheHiveDB image data management and analysis framework. Front. Neuroinform. 7:49. doi: 10.3389/fninf.2013.00049

Rane, P., Haselgrove, C., Hodge, S. M., Frazier, J. A., and Kennedy, D. N. (2014). Structure-centered portal for child psychiatry research. Front. Neuroinform. 8:47. doi: $10.3389 /$ fninf.2014.00047

Rautenberg, P. L., Kumaraswamy, A., Tejero-Cantero, A., Doblander, C., Norouzian, M. R., Kai, K., et al. (2014). NeuronDepot: keeping your colleagues in sync by combining modern cloud storage services, the local file system, and simple web applications. Front. Neuroinform. 8:55. doi: 10.3389/fninf.2014.00055

Sarwate, A. D., Plis, S. M., Turner, J. A., Arbabshirani, M. R., and Calhoun, V. D. (2014). Sharing privacy-sensitive access to neuroimaging and genetics data: a review and preliminary validation. Front. Neuroinform. 8:35. doi: 10.3389/fninf.2014.00035

Sherif, T., Rioux, P., Rousseau, M.-E., Kassis, N., Beck, N., Adalat, R., et al. (2014). CBRAIN: a web-based, distributed computing platform for collaborative neuroimaging research. Front. Neuroinform. 8:54. doi: 10.3389/fninf.2014.00054

Smith, S. M., Jenkinson, M., Woolrich, M. W., Beckmann, C. F., Behrens, T. E., Johansen-Berg, H., et al. (2004). Advances in functional and structural MR image analysis and implementation as FSL. Neuroimage 23(Suppl. 1), S208-S219. doi: 10.1016/j.neuroimage.2004.07.051
Sobolev, A., Stoewer, A., Leonhardt, A., Rautenberg, P. L., Kellner, C. J., Garbers, C., et al. (2014). Integrated platform and API for electrophysiological data. Front. Neuroinform. 8:32. doi: 10.3389/fninf.2014.00032

Tripathy, S. J., Savitskaya, J., Burton, S. D., Urban, N. N., and Gerkin, R. C. (2014). NeuroElectro: a window to the world's neuron electrophysiology data. Front. Neuroinform. 8:40. doi: 10.3389/fninf.2014.00040

Van Horn, J. D., and Toga, A. W. (2009). Neuroimaging workflow design and datamining: a Frontiers in neuroinformatics special issue. Front Neuroinform. 3:31. doi: 10.3389/neuro.11.031.2009

Van Horn, J. D., and Toga, A. W. (2014). Human neuroimaging as a "Big Data" science. Brain Imaging Behav. 8, 323-331. doi: 10.1007/s11682-013-9255-y

Wood, D., King, M., Landis, D., Courtney, W., Wang, R., Kelly, R., et al. (2014). Harnessing modern web application technology to create intuitive and efficient data visualization and sharing tools. Front. Neuroinform. 8:71. doi: 10.3389/fninf.2014.00071

Xiang, J., Luo, Q., Kotecha, R., Korman, A., Zhang, F., Luo, H., et al. (2014). Accumulated source imaging of brain activity with both low and high-frequency neuromagnetic signals. Front. Neuroinform. 8:57. doi: 10.3389/fninf.2014.00057

Zaslavsky, I., Baldock, R. A., and Boline, J. (2014). Cyberinfrastructure for the digital brain: spatial standards for integrating rodent brain atlases. Front. Neuroinform. 8:74. doi: 10.3389/fninf.2014. 00074

Conflict of Interest Statement: The authors declare that the research was conducted in the absence of any commercial or financial relationships that could be construed as a potential conflict of interest.

Copyright $\odot 2015$ Cheng, Marcus, Van Horn, Luo, Mattay and Weinberger. This is an open-access article distributed under the terms of the Creative Commons Attribution License (CC BY). The use, distribution or reproduction in other forums is permitted, provided the original author(s) or licensor are credited and that the original publication in this journal is cited, in accordance with accepted academic practice. No use, distribution or reproduction is permitted which does not comply with these terms. 